\title{
TIFR zero-pressure balloon programme crosses a milestone
}

\section{Anand*, B. Suneel Kumar and Devendra Ojha}

High-altitude scientific balloons offer unique opportunities to carry scientific payloads to stratospheric altitudes at a cost several orders of magnitude lower than the corresponding satellite missions. Balloon-borne payloads are easy to implement allowing quick experiment turn-around times and inexpensive reflights can be conducted as the payload is recovered most of the times. In addition, in situ and high-resolution spatial and temporal measurements of the earth's atmosphere can be made that might not be feasible with satellites. They are also used as a testbed to prove technologies for future satellite missions. Scientific ballooning was initiated at the Tata Institute of Fundamental Research (TIFR), Mumbai in 1945, when scientific instruments were flown to stratospheric altitudes using a cluster of weather balloons for cosmic-ray research. The need to have balloons float at constant stratospheric altitudes for studies in astronomy led to the initiation of work on the design and fabrication of zero-pressure polyethylene (ZP) balloons at TIFR in 1956. Since then, several ZP balloon flights have been conducted for studies in astronomy, atmospheric science, astrobiology, balloon technology and space technology development, leading to several important scientific results. In 2018, the TIFR balloon programme crossed an important milestone of conducting more than $500 \mathrm{ZP}$ balloon flights. This article presents recent advancements made in some areas of scientific ballooning and details of balloon experiments conducted in the past two decades.

Keywords: Cold brittle point, gore, satellite missions, scientific payloads, stratospheric altitudes, zero-pressure balloon.

THE Indian Zero-Pressure polyethylene (ZP) balloon programme started by Tata Institute of Fundamental Research (TIFR), Mumbai, established a permanent balloon facility (BF) at Hyderabad (lat. $17.5^{\circ} \mathrm{N}$, long. $78.5^{\circ} \mathrm{E}$, geomagnetic lat. $8^{\circ} \mathrm{N}$ ) India, in 1969 , with an aim to design, manufacture and launch scientific balloons up to stratospheric altitudes for research in various science disciplines. The scientific advantage of this location is the high cosmic ray vertical cut-off rigidity of $15 \mathrm{GV}$ and easy accessibility to both the northern and southern skies from balloon altitudes, making it ideal for astronomical studies. Several countries worldwide like USA, France, Japan, Australia, Sweden, Italy, Brazil, etc. have balloon programmes and facilities for scientific ballooning. However, $\mathrm{BF}$ is unique in the sense that, it carries out balloon design and manufacture, gondola fabrication and payload integration, provides telecommand, telemetry, tracking (TTC) support and balloon launch - all under one roof, in

D. Anand, B. Suneel Kumar and Devendra Ojha are in the TIFR Balloon Facility, ECIL Post, Hyderabad 500 062, India and Devendra Ojha is also in the Tata Institute of Fundamental Research, Colaba, Mumbai 400005 , India.

*For correspondence. (e-mail: anandd@tifr.res.in) addition to payload recovery. In other words, it provides an end-to-end solution for scientific ballooning. Balloon experiments are conducted not only for TIFR, but also for several national laboratories and universities from India and abroad. BF has the capability to design and manufacture a range of balloon sizes to accommodate various payload masses for the desired float altitude. The ZP balloon flights are conducted on a regional basis due to the restrictions in allocated air corridor by the civil aviation authorities, for balloon flight operations. Therefore, a float duration up to $\sim 10 \mathrm{~h}$ can be achieved based on wind speed and direction at the float altitude. Additionally, a maximum of about $400 \mathrm{~km}$ line-of-sight reliable range of the TTC system puts a limitation on the float duration. In the following sections, we will briefly dwell on balloon technology, support instrumentation, facilities available to scientists, our future plans and focus more on the balloon experiments conducted in the past two decades.

\section{Developments in balloon technology}

To manufacture a reliable ZP balloon for stratospheric altitudes, it is important to have a good balloon film, load 
tapes according to the balloon design, balloon adhesive tape, balloon end fittings and quality control during the balloon manufacture process. The balloon manufacture at $\mathrm{BF}$ is completely indigenous in all these components. Exhaustive details on our early balloons, balloon design and optimized single-cap balloon design are discussed elsewhere ${ }^{1-4}$. Developments made in some of these areas are discussed here.

\section{Indigenous balloon film}

In the mid-eighties, both $\mathrm{M} / \mathrm{s}$ Winzen and Raven, USA, introduced new grades of balloon films, viz. SF-372 and Astrofilm-E2 respectively, with cold brittle point (CBP) around $-96^{\circ} \mathrm{C}$ and exceptional mechanical properties. Inspired by this development, BF extruded balloon-grade film out of 'LADENE $118 \mathrm{~W}$ ' linear low density polyethylene (LLDPE) resin with butene as comonomer having CBP around $-88^{\circ} \mathrm{C}$, and the mechanical properties were lower than SF-372 and Astrofilm-E2. In 1989, balloons using this film were successfully flight-tested. In 1990, superior-grade LLDPE resin 'Dowlex 2045' with octene as comonomer became available in India and the film extruded out of this resin was named Antrix ${ }^{1}$. Laboratory tests of Antrix film revealed its exceptional mechanical properties both at room temperature and at $-80^{\circ} \mathrm{C}$, and CBP of this film was found to be about $-96^{\circ} \mathrm{C}$ (ref. 2). Further improvements were made in the manufacturing process during extrusion leading to better quality of Antrix film. The film is comparable with the best balloon-grade films from USA like SF-372 and Astrofilm-E2. The arrival of Antrix film has been a game changer for scientific ballooning in India, resulting in $100 \%$ success for balloons flown from BF. Currently, all balloons fabricated at $\mathrm{BF}$ are made out of Antrix film. Details on the test methods and tools to determine the mechanical properties of the indigenous Antrix film and comparison with SF-372 and Astrofilm-E2 are discussed elsewhere ${ }^{4}$.

\section{Indigenous load tapes}

During balloon fabrication, balloon film is cut into many banana peel-shaped vertical sections called gores. The gore edges are heat-sealed together along with the load tape made of high-tenacity polyester yarn. Many such gores heat-sealed together form a balloon envelope. During the balloon flight, longitudinal stresses due to suspended load are carried by the load tapes. Therefore, the load tape is the second major and expensive component in balloon manufacture, which was imported in the initial years. A load tape manufacturing unit indigenously developed after years of research and development is fully operational and by 2000 , we were capable of manu- facturing load tapes of breaking strength (BS) up to $136 \mathrm{kgf}$. However, load tapes for heavier infrared astronomy telescope were still being imported. After a compelling search, a polyester yarn of $9.3 \mathrm{~g}$ /denier was identified and a load tape made out of this yarn at BF resulted in a BS of about $182 \mathrm{kgf}$. Balloons made of this load tape proved to be safe to carry the infrared astronomy telescope, making us totally self-reliant ${ }^{5}$. Currently, BF is capable of manufacturing load tape of BS up to $400 \mathrm{kgf}$.

\section{Indigenous balloon adhesive tapes}

Another critical component is the adhesive tape. This is used for reinforcement of the balloon top-end fitting, hydrogen inflation tubes and for carrying out balloon repairs. The adhesive tape that was earlier imported is now manufactured in India. In the initial stages of development, the manufacturer worked closely with the balloon group to customize it for this application. The indigenous tape made out of fluorinated ethylene polypropylene film of thickness $50 \mu \mathrm{m}$ coated with a silicone based adhesive, has all the properties required for balloon applications $^{5}$.

\section{Balloon support instrumentation capabilities}

The balloon flights are supported by high-reliability telecommand uplink for balloon and payload control operations including flight termination, and telemetry downlink for payload health and scientific data. The S-band $3.7 \mathrm{~m}$ Dish TTC ground station provides precise autotracking of the balloon and is capable of handling telemetry data rates up to $1 \mathrm{Mbps}$ in the standard interrange instrumentation group format used in ballooning. A fair amount of redundancy is provided in important subsystems to make flight operations foolproof. Highreliability air safety devices are used to meet compliance with civil aviation rules. Payload is recovered within 1$2 \mathrm{~h}$ of landing due to reliable tracking devices. In the past, custom-designed and experiment-specific on-board electronics was developed to cater to the needs of user scientists. Gondolas of various sizes and shapes were designed and fabricated at BF to provide structural stability to payload during launch as well as landing.

\section{Science and technology-development balloon flights (1999-2018)}

The first ZP balloon flight in India was conducted in 1958 (ref. 3). Up till 2018, 507 ZP balloon flights have been conducted for research in various science disciplines. Balloon flights with gross lifts up to $2500 \mathrm{~kg}$ and payload weight up to $\sim 1000 \mathrm{~kg}$ having dimensions reaching $3 \mathrm{~m}$ diameter and $5.5 \mathrm{~m}$ height can be carried out. 
Currently, the largest balloon that can be manufactured at $\mathrm{BF}$ is $740,000 \mathrm{~m}^{3}$. Payload recovery is almost $100 \%$. Seventy-one successful balloon experiments conducted in various disciplines during this period are briefly discussed here.

\section{$X$-ray astronomy}

A telescope named Large Area Scintillation counter Experiment (LASE) was designed to detect microsecond variations in the flux of X-ray sources in the hard X-ray energy range up to $200 \mathrm{keV}$. Nine balloon flights were conducted during 1999-2012 using this telescope at float altitudes of $41-42 \mathrm{~km}$. Several publications with important results on the rapid variability of galactic and extragalactic X-ray sources in hard X-rays have emerged from these flights ${ }^{6,7}$. Later, the same group developed a new balloon-borne High-Energy X-ray Imaging Telescope (HEXIT) for hard X-ray studies in the energy range 20 $800 \mathrm{keV}$, which was successfully flown in 2005, 2006 and 2009. The second HEXIT payload was launched aloft a balloon of volume 738,900 $\mathrm{m}^{3}$ in the year 2006 (Figure 1). The balloon floated at an altitude of about $41.4 \mathrm{~km}$ for about $1 \mathrm{~h}$ and $21 \mathrm{~min}$, with a suspended load of $938.2 \mathrm{~kg}$ (ref. 8). The third HEXIT flight launched on 9 April 2009 using the same balloon size floated at an altitude of $41.7 \mathrm{~km}$ for about $9 \mathrm{~h}$. This is the second largest balloon

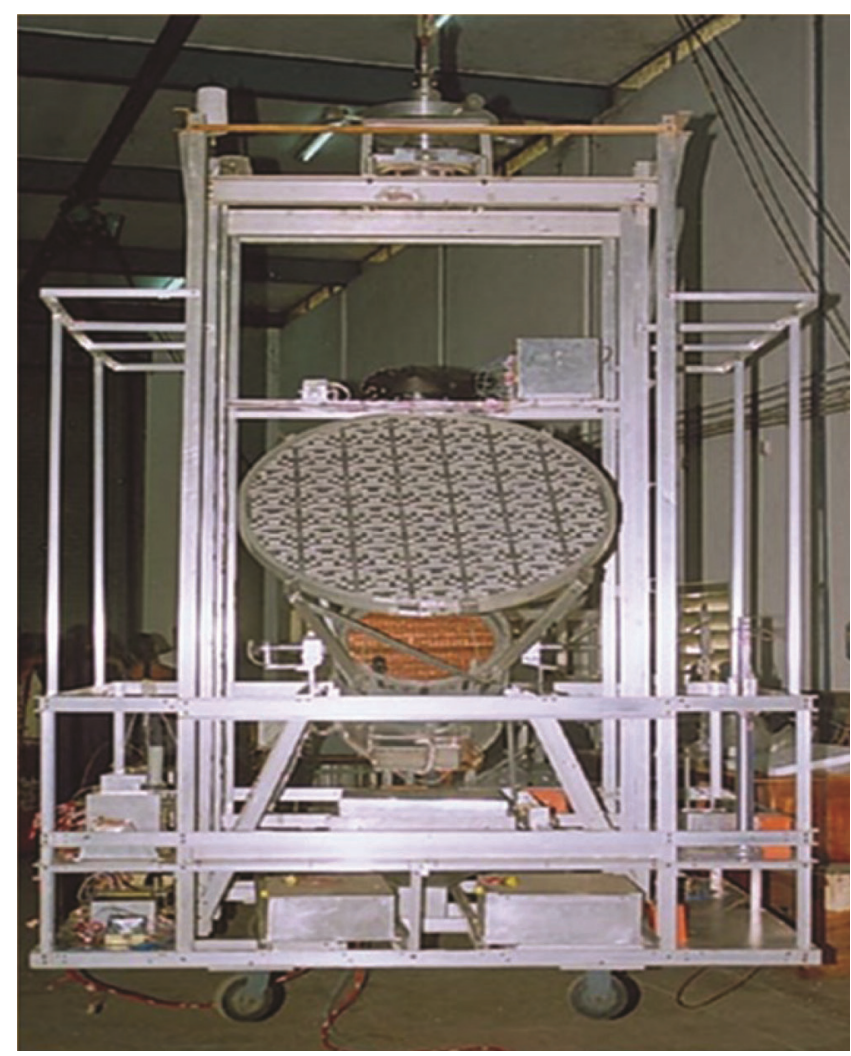

Figure 1. X-ray telescope-HEXIT. designed, fabricated and launched from BF. Another type of X-ray balloon experiment comprising two large area X-ray proportional counters (LAXPCs) was carried out on 14 April 2008. The design of these LAXPCs was similar to those on the AstroSat satellite, except that their field of view was $3^{\circ} \times 3^{\circ}$ compared to $1^{\circ} \times 1^{\circ}$ for LAXPCs on the AstroSat. This experiment was aimed at timing and spectral studies of X-ray sources in the 3-80 keV region. The black hole $\mathrm{X}$-ray binary Cygnus $\mathrm{X}-1$ was observed in the experiment for $\sim 3 \mathrm{~h}$ at a float altitude of about $41 \mathrm{~km}$ (ref. 9). This payload was carried aloft a $739,500 \mathrm{~m}^{3}$ balloon designed and fabricated at BF. This is the largest balloon launched from BF till date, with a suspended load of $954 \mathrm{~kg}$. The experiments described were conducted by the TIFR X-ray astronomy group.

\section{Infrared astronomy}

As a part of the TIFR-Japan collaboration in balloonborne Far Infra-Red (FIR) astronomy, a Fabry-Perot Spectrometer (FPS) developed by the Institute of Space and Astronautical Science, Japan, was successfully installed at the focal plane of the TIFR $100-\mathrm{cm}$ balloon-borne telescope. This new telescope combination henceforth is referred to as FPS100 (Figure 2). The FPS (R 1800) is tuned to the astrophysically interesting fine structure line

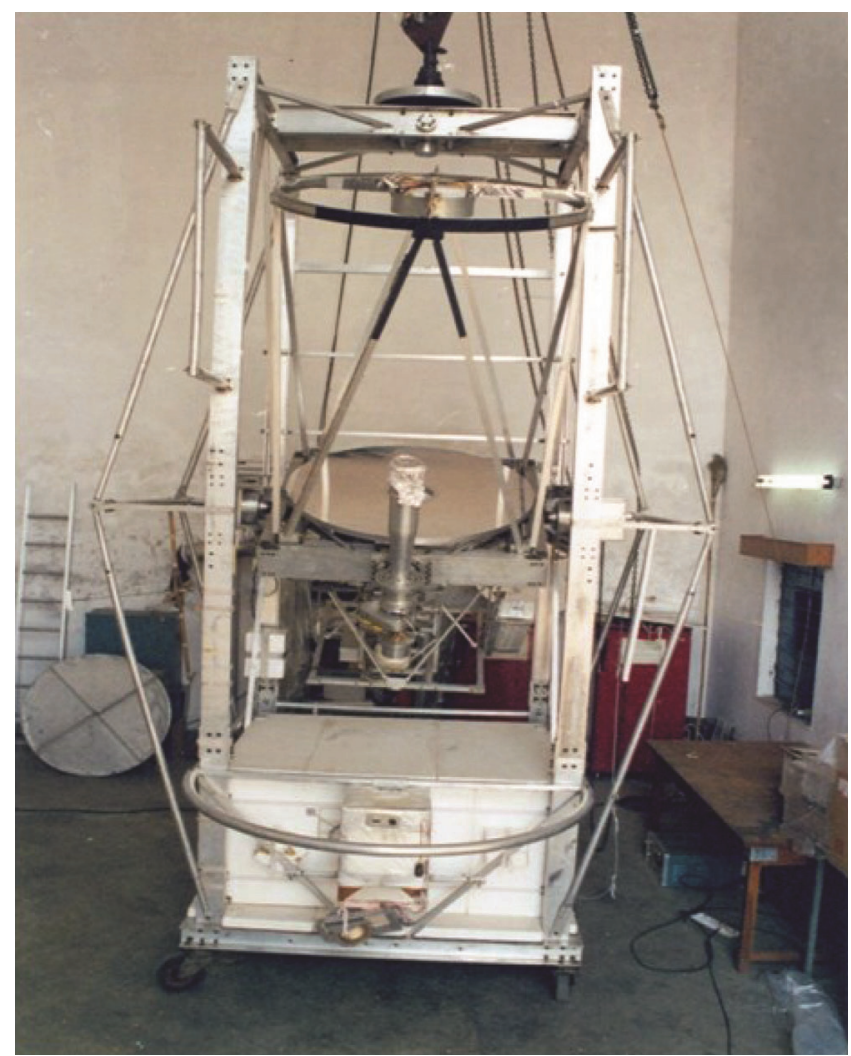

Figure 2. FPS100-FIR telescope. 
of (C II) at $157.74 \mu \mathrm{m}$. (C II) $158 \mu \mathrm{m}$ line is a major gas-cooling channel of the neutral gas-phase interstellar medium (ISM), and thus is a key to understanding ISM properties influenced by the radiative feedback of massive stars. The first successful flight of FPS100 was conducted on 25 November 1999 (ref. 10). Till date, FPS100 weighing $\sim 1080 \mathrm{~kg}$ with ballast has been successfully flown 10 times since its first launch. As a result of recent continuous successful observations, unprecedented large-area $(\sim 15 \operatorname{arcmin} \times 30$ arcmin $)$ (C II) maps of galactic carbon line were made from several galactic massive star-forming regions (viz. RCW 38, Orion Bar, W3, RCW 36, NGC 2024, W31, Carina, NGC 6334 and NGC 6357) ${ }^{11}$. Under another TIFR-Japan collaboration, five successful flights were conducted from 2000 to 2003 using the Japanese Far Infra-Red Balloon Experiment (FIRBE) telescope. FIRBE developed mainly by the Japanese Nagoya University team and partly by the TIFR Infrared Astronomy Group, has a 50-cm off-axis paraboloidal mirror at the focus of which a 32-element mechanically stressed photoconductor detector array at $155 \mu \mathrm{m}$ is mounted. Interesting results were obtained from the widearea mapping of the $155 \mu \mathrm{m}$ continuum emission in the Orion molecular cloud complex ${ }^{12}$.

\section{Atmospheric science and astrobiology}

The period under discussion has seen a revival of balloonborne experiments for atmospheric science research. Many of them are first of their kind experiments from BF.

A low-power, high-pulse repetition frequency laserbased micro pulse lidar operating at $532 \mathrm{~nm}$ having $150 \mathrm{~mm}$ Cassegrain-type telescope and a photomultiplier tube-based photon-counting data acquisition system was developed by Indian Space Research Organisation (ISRO) for Balloon-Borne Lidar (BBL)-based measurement of backscattered signal from aerosols and clouds. This downward-looking $459 \mathrm{~kg}$ BBL was successfully launched using a $109,700 \mathrm{~m}^{3}$ balloon on 16 April 2009 at 00:36 IST (ref. 13). The flight was terminated after a float duration of $2 \mathrm{~h}$ at $35 \mathrm{~km}$ altitude. This was the first time that a BBL was flown up to stratospheric altitude from BF.

An Indo-US Balloon-borne Investigation of Regionalatmospheric Dynamics (BIRD) Experiment was conducted on 8 March 2010 . The $109,755 \mathrm{~m}^{3}$ balloon carrying a $335 \mathrm{~kg}$ BIRD payload reached a float altitude of about $35 \mathrm{~km}$ at 12:45 IST. The flight was terminated at 18:25 IST. The gondola orientation, designed by BF, was programmed to rotate in the azimuth direction in a unique fashion to achieve the experimental objectives. The pointing accuracy of the orientation platform attained was 20 arcmin. This was perhaps the first daytime balloonborne optical investigation carried out on mesospherelower troposphere wave dynamics ${ }^{14}$. This was also the first time that a balloon was launched around noon from BF.
An aethalometer (model AE-42, Magee Scientific, USA) was flown aloft a balloon of volume $109,755 \mathrm{~m}^{3}$ on 17 March 2010. The payload weighing $350 \mathrm{~kg}$ reached a float altitude of $35 \mathrm{~km}$. The balloon ascent rate for this flight was maintained at $\sim 2.6 \mathrm{~m} / \mathrm{s}$ to enable highresolution vertical profiles. This was the first ever in situ measurement of black carbon aerosols in the troposphere (up to $9 \mathrm{~km}$ ) made over central India and was conducted by the Space Physics Laboratory, Thiruvananthapuram ${ }^{15}$. The same experiment was repeated twice later in 2011 for further investigations.

A Balloon Experiment on the Electrodynamics of Near Space (BEENS) was launched using a $110,000 \mathrm{~m}^{3}$ balloon on 14 December 2013. The balloon reached a float altitude of $35.2 \mathrm{~km}$ and the flight was terminated after $4 \mathrm{~h}$ of float duration. The payload comprising four deployable booms for measurements of horizontal electric fields and one inclined boom for vertical electric field measurement was successfully deployed through telecommand after launch ${ }^{16}$. A unique orientation mechanism for the experiment was designed by the BF engineers. The experiment was conducted by the Indian Institute of Geomagnetism, Navi Mumbai.

The Balloon measurement campaigns of the Asian Tropopause Aerosol Layer (BATAL), is a NASA-ISROsponsored campaign in India and elsewhere to study the nature, formation and transport of polluted aerosols in the upper troposphere and lower stratosphere during the Asian summer monsoon season ${ }^{17}$. BATAL ZP balloon flights were conducted in 2015 and 2017-2019 from BF. This was the first time that the balloons were designed and fabricated by BF for the upper troposphere and lower stratosphere float altitudes and medium payload mass. The on-board telemetry, telecommand, GPS interface, air-safety transponder and batteries were customdesigned by BF to keep the weight as low as possible $(11 \mathrm{~kg})$. A miniaturized ballast system was designed that helped achieve the balloon to float about the tropopause region. This was also the first time that $\mathrm{ZP}$ balloon launches were conducted during the Indian summer monsoon season when high surface winds prevail, making the launch challenging. Special permission was sought from the Airport Authority of India due to the proximity of the balloon float altitude to aircraft cruise altitude $(\sim 12 \mathrm{~km})$.

Three balloon-borne experiments were conducted in the area of astrobiology that involved many astronomers and biologists. The cryogenic sampler payload was developed by ISRO. The first one was launched on 29 April 1999, the second on 21 January 2001 and the third on 20 April 2005. Air samples were collected aseptically at altitudes of 20-41 km using a cryogenic sampler comprising 16-cryoprobe assembly, for studies on the qualitative and quantitative distribution of microorganisms in the upper troposphere-stratosphere. Few new and novel bacterial strains were found in the samples collected ${ }^{18,19}$. 


\section{Balloons for mesospheric altitudes}

Work on the development of an ultra-thin balloon grade polyethylene film, using m-LLDPE resin with added metallocene catalyst, in the thickness range $2.8-3.8 \mu \mathrm{m}$ for fabricating high-altitude balloons capable of penetrating the mesosphere commenced in 2011 to meet the needs of scientists working in the area of atmospheric dynamics. After an initial setback in 2012, three flights were conducted in 2014, using $61,013 \mathrm{~m}^{3}$ balloons made out of $3.8 \mu \mathrm{m}$ film with a suspended load of about $10 \mathrm{~kg}$. All the three balloons crossed into the mesosphere reaching altitudes of over $51 \mathrm{~km}$; the highest altitude reached was $51.83 \mathrm{~km}$ (ref. 20).

\section{Balloons for StratEx human spaceflight record}

On 24 October 2014, Alan Eustace, USA, ascended to an altitude of $41.578 \mathrm{~km}$ under a $328,232 \mathrm{~m}^{3}$ balloon - the greatest height a person has ever reached without rocket propulsion. As the balloon descended to an altitude of $41.422 \mathrm{~km}$, Eustace detached himself from the balloon with the aid of a pyrocutter device and plummeted toward the earth at speeds that peaked at $821 \mathrm{miles} / \mathrm{h}$, creating a new world altitude record for a skydive, which stands unbroken till date. The balloon for this flight was designed and manufactured at BF using indigenous Antrix film and was launched by the StratEx team from New Mexico, USA. According to StratEx team (Nott et al. ${ }^{21)}$, 'The team feel that TIFR's proprietary films and balloon assembly are of the highest standard and had no qualms about the safety of launching Alan Eustace under these balloons'.

\section{Animal spaceflight}

A capsule with three live lab rats with life-support system for its survival was launched aloft a $39,915 \mathrm{~m}^{3}$ balloon on 14 March 2015. The flight was terminated after the balloon reached an altitude of $29.5 \mathrm{~km}$. The design of lifesupport system was successful as all the rats were recovered alive and healthy. BF engineers contributed in making the capsule leak-proof. The experiment was carried out by InGenius, Singapore - the company behind the project. This was the first time such an experiment was conducted from BF.

\section{Engineering test flight - 500th flight (T500)}

The FPS100 telescope, which was in the process of being upgraded, required enhanced telemetry data-handling capability. After considering several options, it was felt that the easiest way is to split the data and transmit them through two independent radio-frequency (RF) transmis- sions. Currently, all telemetry data come through a single RF transmission. The on-board electronics was designed to transmit two telemetry data on two different frequencies, one at the standard $10 \mathrm{kbs}$ data rate on $2259 \mathrm{MHz}$ and the other at $250 \mathrm{kbps}$ on $2281 \mathrm{MHz}$. In order to flight-test the dual-telemetry scheme and several other electronics, an engineering test flight was conducted on 13 April 2018. The balloon of volume $3026 \mathrm{~m}^{3}$ with a suspended load of $72 \mathrm{~kg}$ reached a float altitude of $24.9 \mathrm{~km}$. The flight was terminated after float duration of $3 \mathrm{~h} 20 \mathrm{~min}$. Figure 3 shows the T500 balloon before liftoff. A BF-customized six-stage Quartz Crystal Microbalance (QCM; California Measurements, USA) impactor, which was first flown on 7 May 2016 (ref. 22), was again flown in this flight to make aerosol measurements in the free troposphere at different altitudes. The telemetry was received by a common dish antenna, followed by two independent chains of data-acquisition equipment in the control room. The performance of the dual-telemetry scheme, QCM, including new on-board electronics was satisfactory. QCM data are being investigated. This was the first time that the dual-telemetry scheme was successfully flight-tested and is likely to open up new opportunities for complex balloon experiments. As a coincidence, on this date, the TIFR balloon programme reached an important milestone of conducting $500 \mathrm{ZP}$ balloon flights in India.

Figure 4 shows the balloon flights conducted yearwise. Figure 5 shows the percentage distribution by various discipline.

\section{Facilities}

$\mathrm{BF}$ has a $1 \mathrm{~m}$ dia. $\times 1.5 \mathrm{~m}$ long thermal-vacuum chamber that operates in the temperature range of $-40^{\circ} \mathrm{C}$ to $80^{\circ} \mathrm{C}$; a vacuum level of $3.3 \times 10^{-5} \mathrm{mb}$ can be achieved and a

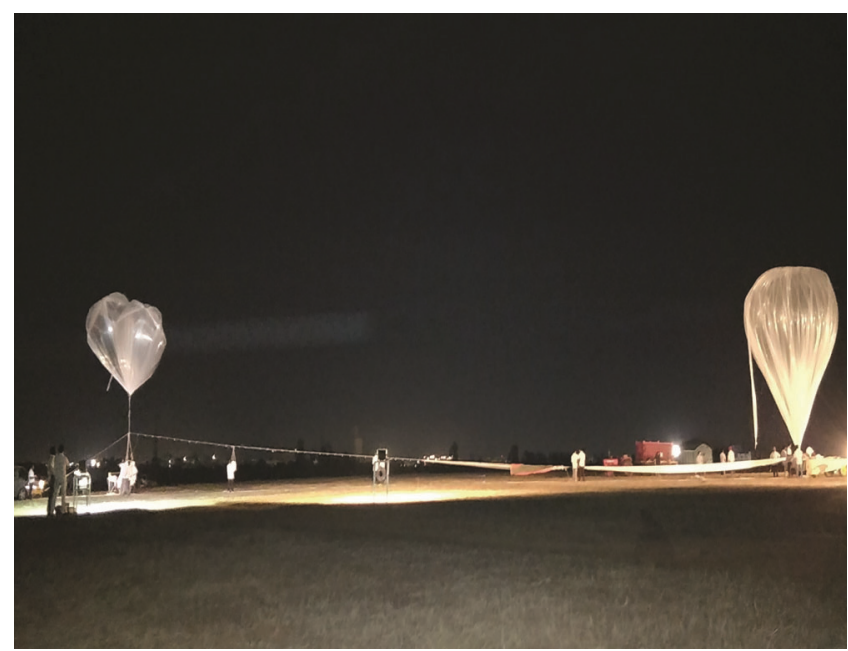

Figure 3. Hydrogen-inflated $\mathrm{T} 500$ balloon before lift-off-static anchor line launch method. 
programmable hot chamber can attain $+100^{\circ} \mathrm{C}$. For calibration of pointing system, a non-magnetic suspension stand, two clean-room facilities of class 10,000, four laminar air-flow benches of class 100 and a shaker for shock and vibration test are also available. There are four laboratories for scientists to work on their payloads and a rigging area for payload tests. A mechanical workshop and electronics test and measurement equipment are available for use to the scientists. Guest house and canteen facility are available within the campus.

\section{Future plans}

As payloads are becoming more complex and heavier, design and development of ZP balloons that can carry payloads heavier than $1000 \mathrm{~kg}$ to altitudes of $38 \mathrm{~km}$ and above has been initiated. Currently, ZP balloons designed by $\mathrm{BF}$ can carry payloads of $\sim 1000 \mathrm{~kg}$ to an altitude of $32 \mathrm{~km}$. A ZP balloon is under development that can carry about $5000 \mathrm{~kg}$ payload to a float altitude of $14-15 \mathrm{~km}$. These balloons find applications in testing the performance

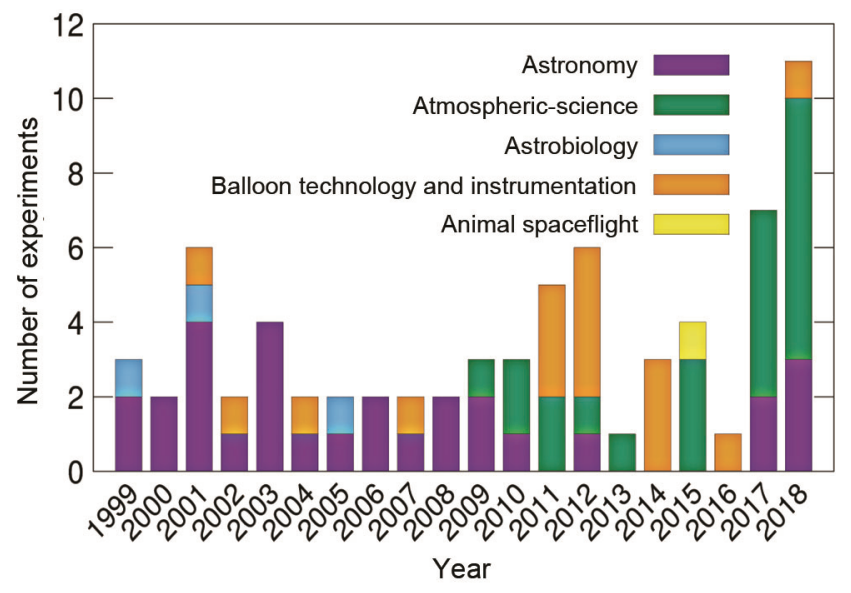

Figure 4. Balloon experiments conducted yearwise from 1999 to 2018.

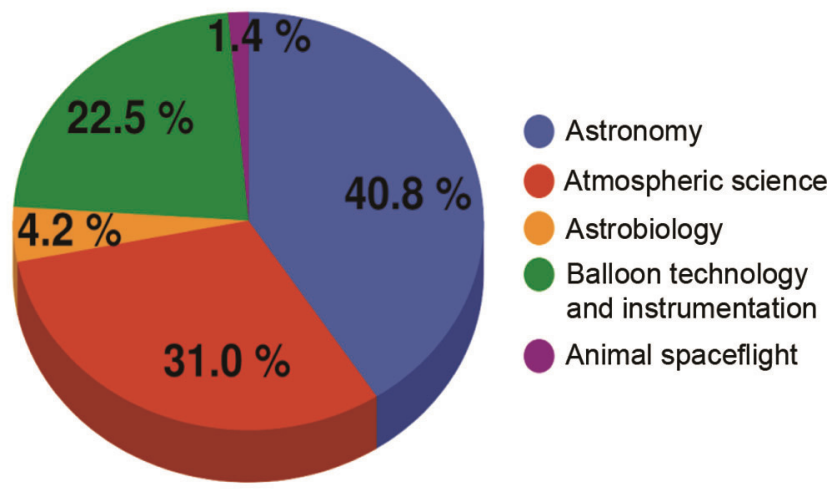

Figure 5. Percentage distribution of balloon experiments from 1999 to 2018 . of re-entry capsules of human space-flight missions. In recent times there has been an increasing demand for tethered kytoons capable of hoisting $20-25 \mathrm{~kg}$ payloads at $300 \mathrm{~m}$ altitude for testing communication payloads and to deploy internet services in remote areas for disaster management. To cater to these needs of national importance, design and fabrication of $100 \mathrm{~m}^{3}$ kytoon using a dual-layer polyethylene film is under progress. We are pursuing development of Super-Pressure (SP) balloons, as there is a growing need from the scientific community for long and ultra-long duration flights. A suitable balloon film has been developed, and design and manufacture of SP balloons is in progress. The scientific ballooning community (from USA, Japan, France, Australia, etc.) has established necessary infrastructure at several launch bases spread across the world (Antarctica, Australia and Sweden - just to name a few), and has been conducting long-duration flights, using ZP and SP balloons, ranging from a few days to several weeks. In collaboration with these countries and with substantial funding, TIFR can implement such missions in the future.

\section{Proposal for balloon experiments}

Proposal for balloon experiments can be sent to the scientist-in-charge over e-mail (our website is https://www. tifr.res.in/ bf/). After examining the feasibility, further course of action will be communicated. A soft copy of the $\mathrm{BF}$ user's manual is available on request.

\section{Conclusion}

The importance of scientific ballooning for research in astronomy, atmospheric science, astrobiology and development of space technology is demonstrated beyond doubt. Several first of their kind balloon experiments were conducted from BF. Payload recovery facilitated multiple reflights to be conducted leading to important scientific observations and results. Several national and international research institutions have conducted balloon-borne experiments from BF. The X-ray detector which was tested on a balloon platform before being successfully deployed in AstroSat-a first dedicated Indian astronomy satellite mission - is providing excellent scientific results. During the past two decades, there have been major innovations made in all areas of scientific ballooning, making BF self-reliant. New initiatives to design and develop large kytoons, SP balloons for long duration flights and ZP balloons to carry heavier payloads will provide new opportunities to do scientific research and test space technologies.

1. Redkar, R. T., Scientific ballooning in India. Indian J. Radio Space Phys., 1991, 20, 169-175. 
2. Damle, S. V. and Joshi, M. N., Developments and advances in scientific balloon capability at the TIFR National Balloon Facility, Hyderabad. Adv. Space Res., 1998, 21(7), 941-948.

3. Gokhale, G. S., Menon, M. G. K. and Redkar, R. T., Stratospheric flights over tropical latitudes with polyethylene balloons of large volume. Proc. Indian Acad. Sci. Sect. A, 1966, 64(2), 57-63.

4. Suneel Kumar, B. et al., Mechanical properties of ANTRIX balloon film and fabrication of single cap large volume balloons. $A d v$. Space Res., 2008, 42, 1691-1697.

5. Manchanda, R. K., Scientific ballooning in India. Adv. Space Res., 2006, 37, 2015-2020.

6. Manchanda, R. K., High energy X-ray observations of NGC 5506 Adv. Space Res., 2006, 38, 1387-1392.

7. Manchanda, R. K., Characteristics and performance of thin $\mathrm{LaBr} 3(\mathrm{Ce})$ crystal for hard X-ray astronomy. Adv. Space Res., 2011, 47, 30-36.

8. Manchanda, R. K., Current developments and future plans at NBF@Hyderabad and prospects for long duration balloon flights. Trans. JSASS Aerospace Technol. Jpn, 2010, 8(27), 5562 .

9. Roy, J. et al., Performance of large area X-ray proportional counters in a balloon experiment. Exp. Astron., 2016, 42(2), 249-270.

10. Mookerjea, B. et al., Mapping of large scale $158 \mu \mathrm{m}$ (CII) line emission: Orion A. Astron. Astrophys., 2003, 404(2), 569-578.

11. Kaneda, H. et al., Large-scale mapping of the massive starforming region RCW38 in the (CII) and PAH emission. Astron. Astrophys., 2013, 556, A92.

12. Arimura, S. et al., Wide-area mapping of 155 micron continuum emission from the Orion molecular cloud complex. Publ. Astron. Soc. Jpn., 2004, 56(1), 51-60.

13. Presenna Kumar, B. et al., High altitude balloon borne Lidar experiment for the study of aerosols and clouds. COSPAR, 2010, 38, 4055.

14. Pallamraju, D. et al., Day time wave characteristics in the mesosphere lower thermosphere region: results from the balloon-borne investigations of regional-atmospheric dynamics experiment. J. Geophys. Res. Space Phys., 2014, 119, 2229-2242.

15. Suresh Babu, S. et al., Free tropospheric black carbon aeroso measurements using high altitude balloon: Do BC layers build 'their own homes' up in the atmosphere? Geophys. Res. Lett., 2011, 38, L08803.
16. Gurubaran, S. et al., A high-altitude balloon experiment to probe stratospheric electric fields from low latitudes. Ann. Geophys., 2017, 35, 189-201.

17. Vernier, J. P. et al., The balloon measurement campaigns of the Asian tropopause aerosol layer. Bull. Am. Meterol. Soc., 2018, 99(5), 955-973.

18. Narlikar, J. V. et al., A balloon experiment to detect microorganisms in the outer space. Astrophys. Space Sci., 2003, 285, 555562.

19. Shivaji, S. et al., Janibacter hoylei sp. nov., Bacillus isronensis sp. nov. and Bacillus aryabhattai sp. nov., isolated from cryotubes used for collecting air from the upper atmosphere. Int. J. Syst. Evolution. Microbiol., 2009, 59, 2977-2986.

20. Suneel Kumar, B. et al., Development of ultra-thin polyethylene balloons for high altitude research up to mesosphere. J. Astronom. Instrum., 2014, 3(2), 1440002.

21. Nott, J. et al., Balloon aspects of StratEx world altitude record sky dive. In AIAA Balloon Systems Conference, Dallas, TX, USA, 22-26 June 2015.

22. Sinha, P. R. et al., Development of balloon-borne impactor payload for profiling free tropospheric aerosol. Aerosol Sci. Technol., 2019, 53(3), 1-38.

ACKNOWLEDGEMENTS. The TIFR balloon programme is greatly indebted to Dr Homi J. Bhabha, Prof. M. G. K. Menon, Prof. G. S. Gokhale, Prof. Bernard Peters, Prof. R. R. Daniel, Mr R. T. Redkar, Prof. S. V. Damle and Mr M. N. Joshi, who pioneered the art and science of scientific ballooning in India. We thank Prof. P. C. Agrawal, Prof. R. K. Manchanda, Prof. S. K. Ghosh and Mr S. Srinivasan for their major contributions. We also thank our colleagues at the TIFR Balloon Facility, Hyderabad for their dedicated support, and the Department of Atomic Energy, Government of India, for support under Project Identification No. RTI 4002. We thank the anonymous reviewer for useful comments and suggestions.

Received 15 May 2020; revised accepted 22 March 2021

doi: $10.18520 / \mathrm{cs} / \mathrm{v} 120 / \mathrm{i} 11 / 1672-1678$ 